\title{
SIMILAR POWERS OF A MATRIX
}

\author{
GERALD BOURGEOIS
}

\begin{abstract}
Let $p, q$ be coprime integers such that $|p|+|q|>2$. We characterize the matrices $A \in \mathcal{M}_{n}(\mathbb{C})$ such that $A^{p}$ and $A^{q}$ are similar. If $A$ is invertible, we prove that $A$ is a polynomial in $A^{p}$ and $A^{q}$. To achieve this, we study the matrix equation $B^{-1} A^{p} B=A^{q}$. We show that for such matrices, $B^{-1} A B$ and $A$ commute. When $A$ is diagonalizable, $A$ is a root of $I_{n}$ and $B^{-1} A B$ is a power of $A$. We explicitly solve the previous equation when $A$ has $n$ distinct eigenvalues or when $A$ has a sole eigenvalue. In the second part, we completely solve the $2 \times 2$ case of the more general matrix equation $A^{r} B^{s} A^{r^{\prime}} B^{s^{\prime}}= \pm I_{2}$.
\end{abstract}

\section{INTRODUCTION}

In [1], the matrices $A \in \mathcal{M}_{n}(\mathbb{C})$ such that $A^{p}=A$, with $p \geq 3$, are characterized. In particular, it is shown that such an equality holds if and only if $A^{\#}=A^{p-2}$ where $A^{\#}$ is the group inverse of $A$.

In [4, the authors deal with a $\{K, s+1\}$-potent matrix, that is, a matrix $A$ such that $K A^{s+1} K=A$, where $s \geq 1$ and $K$ is an involutory matrix. Clearly $A^{s+1}$ and $A$ are similar. They prove the following result

Theorem. [4, Theorem 5] Let $A \in \mathcal{M}_{n}(\mathbb{C}),\left\{\lambda_{1}, \cdots, \lambda_{t}\right\}$ be the spectrum of $A$ and, for every $j \leq t$, let $P_{j}$ be the eigenprojection associated to $\lambda_{j}$. The matrix $A$ is $\{K, s+1\}$-potent if and only if $A^{(s+1)^{2}}=A$ and, for every $i \leq t$, there exists a unique $j \leq t$ such that $\lambda_{i}=\lambda_{j}^{s+1}$ and $P_{i}=K P_{j} K$.

More related results can be found in [5, 7, 8, or in [6].

In the first part of this paper, we are interested in a more general problem: let $p, q$ be coprime integers such that $|p|+|q|>2$. We consider the $n \times n$ complex matrices $A$ such that $A^{p}$ and $A^{q}$ are similar. Clearly, if $A^{p}$ and $A^{q}$ are similar, then these two matrices have the same spectrum. The converse is false as we can see it in the following example

$$
A=\operatorname{diag}\left(\left(\begin{array}{cc}
i & 0 \\
0 & i
\end{array}\right),\left(\begin{array}{cc}
-i & 1 \\
0 & -i
\end{array}\right), J_{3}\right)
$$

where $J_{3}$ is the Jordan nilpotent block of dimension 3. The matrices $A^{3}$ and $A^{5}$ have the same spectrum but are not similar. However $A^{3}$ and $A^{7}$ are similar. Assume that $0<p<q$ and that $A^{p}$ and $A^{q}$ are similar. We can easily show that there exists $k \leq n$ such that $A$ is similar to $\operatorname{diag}(N, T)$ where $N \in \mathcal{M}_{k}(\mathbb{C})$ satisfies $N^{p}=0_{k}$ and $T \in G L_{n-k}(\mathbb{C})$ is such that $T^{p}$ and $T^{q}$ are similar. In the particular case where $A^{p}=A^{q}, A^{p}$ is diagonalizable. This conclusion is not true in general, as we can check it in the previous example.

In Theorem 1, we characterize the matrices $A$ such that $A^{p}$ and $A^{q}$ are similar. In this case, every eigenvalue of $A$ is shown to be 0 or a root of unity. In the case when $A$ is invertible, $A$ is a polynomial in $A^{p}$ or in $A^{q}$. More explicitly, we consider

2010 Mathematics Subject Classification. Primary 15A30, Secondary 15A24.

Key words and phrases. Matrix equation, Similar matrices, Powers of matrices. 
the matrix equation

$$
B^{-1} A^{p} B=A^{q}
$$

where the $n \times n$ complex invertible matrices $A, B$ are to be determined.

We show that the matrices $B^{-1} A B$ and $A$ commute. Moreover if $A$ is diagonalizable, then $A$ is a root of $I_{n}$ and $B^{-1} A B$ is a power of $A$. In Theorem 2, we completely solve Eq. (1) when $A$ has $n$ distinct eigenvalues. Finally, the case where $A$ has a sole eigenvalue is considered too.

In the second part of the article, we have a look at the $2 \times 2$ case of a generalization of Eq. (1). Let $\epsilon \in\{-1,1\}$ and let $r, r^{\prime}, s, s^{\prime}$ be given non-zero integers such that $\operatorname{gcd}\left(r, r^{\prime}\right)=1$ and $\operatorname{gcd}\left(s, s^{\prime}\right)=1$. We consider the matrix equation

$$
A^{r} B^{s} A^{r^{\prime}} B^{s^{\prime}}=\epsilon I_{n}
$$

We reduce the problem to the case where $A$ and $B$ are in $S L_{2}(\mathbb{C})$ and are not simultaneously triangularizable. In Theorem 3 the solutions of Eq. (2) are given. Moreover, if $r \neq r^{\prime}$ and $s \neq s^{\prime}$, then $A, B$ and $A^{r} B^{s}$ are roots of $I_{2}$. The methods employed to solve Eq. (2) are essentially different from those employed to solve Eq. (1).

We introduce notations that will be used in the sequel of the article.

Notation. $i$ ) Denote by $\mathbb{N}$ the set of positive integers.

ii) If $A$ is a square complex matrix, then $\sigma(A)$ denotes the set of distinct eigenvalues of $A$ and $m \sigma(A)$ denotes the set of eigenvalues of $A$ with multiplicities.

iii) A multiset $U$ is a set of complex numbers with multiplicities. Moreover, if $p \in \mathbb{Z}, U^{p}$ denotes the multiset of the $p$-th powers of the elements from $U$ with multiplicities.

\section{Similarity of $A^{p}$ AND $A^{q}$}

We consider the matrix equation

$$
B^{-1} A^{p} B=A^{q}
$$

when $A, B \in G L_{n}(\mathbb{C})$ and $p, q$ are given coprime integers. To avoid trivial situations, we assume that $|p|+|q|>2$. In the sequel, we put $C=B^{-1} A B$.

Proposition 1. Let $U$ be some finite multiset of non-zero complex numbers. Assume that $U^{p}$ and $U^{q}$ are equal. The following assertions hold

i) Elements of $U$ are roots of unity. Moreover their orders are coprime to pq.

ii) If $\lambda, \mu \in U$ are such that $\lambda^{p}=\mu^{p}$ or $\lambda^{q}=\mu^{q}$, then $\lambda=\mu$.

iii) For every $\lambda_{1} \in U$ there exists a unique sequence $\left(\lambda_{i}\right)_{i \in \mathbb{Z}}$ of elements of $U$ with, for every $i \in \mathbb{Z}, \lambda_{i}^{q}=\lambda_{i+1}^{p}$.

iv) The application $\left(k, \lambda_{1}\right) \in \mathbb{Z} \times U \rightarrow \lambda_{k+1} \in U$ is an action of $\mathbb{Z}$ on $U$. The order of each element of the orbit $O_{\lambda_{1}}$ of $\lambda_{1}$ divides $p^{r_{1}}-q^{r_{1}}$ where $r_{1}=\operatorname{card}\left(O_{\lambda_{1}}\right)$.

Proof. $i)$ Let $\lambda_{1} \in U$. There exist $\lambda_{2} \in U$ such that $\lambda_{1}^{q}=\lambda_{2}^{p}$ and $\lambda_{0} \in U$ such that $\lambda_{0}^{q}=\lambda_{1}^{p}$. Inductively we can build a sequence $\left(\lambda_{i}\right)_{i \in \mathbb{Z}}$ in $U$ with $\lambda_{i}^{q}=\lambda_{i+1}^{p}$ for all $i \in \mathbb{Z}$. Since $U$ is finite, there exist positive integers $u, v(u<v)$ such that $\lambda_{u}=\lambda_{v}$ and such that, for $u_{1}<v_{1}<v$, one has $\lambda_{u_{1}} \neq \lambda_{v_{1}}$. Since $\lambda_{1}^{q^{u-1}}=\lambda_{u}^{p^{u-1}}$ and $\lambda_{1}^{q^{v-1}}=\lambda_{v}^{p^{v-1}}$, we obtain that

$$
\lambda_{1}^{q^{u-1} p^{v-u}}=\lambda_{u}^{p^{v-1}}=\lambda_{1}^{q^{v-1}} .
$$

Hence, $\lambda_{1}$ is a root of unity of order $k$ dividing $q^{u-1}\left(p^{v-u}-q^{v-u}\right)$. Thus $k$ and $p$ are coprime. In the same way, $k$ and $q$ are coprime. Finally $k$ divides $p^{v-u}-q^{v-u}$. ii) Now if $\lambda \neq \mu$ and $\lambda^{q}=\mu^{q}$, we have $(\lambda / \mu)^{q}=1$. Therefore the order of $\lambda / \mu$ 
is a divisor of $q$ which is $>1$. On the other hand, the order of $\lambda / \mu$ divides the least common multiple of the orders of $\lambda$ and $\mu$, and this number is coprime to $p q$. Hence, we obtain a contradiction. Therefore $\lambda=\mu$.

iii) By $i)$ and $i i)$, the sequence $\left(\lambda_{i}\right)_{i \in \mathbb{Z}}$ is well defined.

$i v$ ) Since $\lambda_{1}^{p^{v-u}}=\lambda_{1}^{q^{v-u}}=\lambda_{1+v-u}^{p^{v-u}}$, we have $\lambda_{1+v-u}=\lambda_{1}$ and $u=1$. We deduce that $\left\{\lambda_{1}, \cdots, \lambda_{v-1}\right\}$ is the orbit of $\lambda_{1}$.

Remark 1. $i$ ) When $U$ contains $r$ copies of the element $\lambda_{1}$, we associate $r$ orbits equal to $O_{\lambda_{1}}$. The elements of $U$ that are in the same orbit have the same order of multiplicity.

ii) Let $V$ be the set of distinct elements of $U$. The action of $\mathbb{Z}$ induces a permutation $\pi$ of $V$. If $\delta$ is the lcm of the cardinalities of the orbits, then the order of each element of $U$ divides $p^{\delta}-q^{\delta}$ and $\delta$ is the order of $\pi$.

Lemma 1. Let $N, M \in \mathcal{M}_{n}(\mathbb{C})$ be nilpotent matrices and $\lambda, \mu \in \mathbb{C}^{*}$. Then $\left(\lambda I_{n}+\right.$ $N)^{p}$ and $\left(\mu I_{n}+M\right)^{q}$ are similar if and only if $\lambda^{p}=\mu^{q}$ and the matrices $N, M$ are similar.

Proof. An easy calculation gives

$$
\left(\lambda I_{n}+N\right)^{p}=\lambda^{p} I_{n}+p \lambda^{p-1} N Z
$$

where $Z$ is an invertible matrix such that $Z N=N Z$. Thus for every $k \in \mathbb{N}$,

$$
\operatorname{ker}\left(\left(\left(\lambda I_{n}+N\right)^{p}-\lambda^{p} I_{n}\right)^{k}\right)=\operatorname{ker}\left(N^{k}\right) .
$$

If $M, N$ are similar, then, for every $k \in \mathbb{N}, \operatorname{dim}\left(\operatorname{ker}\left(M^{k}\right)\right)=\operatorname{dim}\left(\operatorname{ker}\left(N^{k}\right)\right)$ and the characteristic spaces of $\left(\lambda I_{n}+N\right)^{p}$ and $\left(\mu I_{n}+M\right)^{q}$ have the same dimension.

Notation. Let $A \in G L_{n}(\mathbb{C})$.

i) Its decomposition in Jordan normal form can be written

$$
A=P \operatorname{diag}\left(\lambda_{1} I_{i_{1}}+N_{1}, \cdots, \lambda_{m} I_{i_{m}}+N_{m}\right) P^{-1}
$$

where $P$ is an invertible matrix, $\sigma(A)=\left(\lambda_{k}\right)_{k \leq m}$ (the $\left(\lambda_{k}\right)_{k \leq m}$ are pairwise distinct and $i_{k}$ denotes the multiplicity of $\left.\lambda_{k}\right)$ and $\left(N_{k}\right)_{k \leq m}$ are Jordan nilpotent matrices. ii) If moreover $(m \sigma(A))^{p}=(m \sigma(A))^{q}$, by Proposition 1 $\left.i v\right)$, for $U=m \sigma(A)$, we can associate the set of distinct orbits $\left(O_{j}\right)_{j \leq \tau}$ and their multiplicities $\left(r_{j}\right)_{j \leq \tau}$.

From the previous results, we deduce easily

Theorem 1. Let $A \in \mathcal{M}_{n}(\mathbb{C})$ such that $(m \sigma(A))^{p}=(m \sigma(A))^{q}$.

i) When $A$ is invertible, the matrices $A^{p}$ and $A^{q}$ are similar if and only if for every $j \leq \tau, \lambda_{k}, \lambda_{l} \in O_{j}$, one has $i_{k}=i_{l}=r_{j}$ and $N_{k}=N_{l}$ up to orderings of the Jordan nilpotent blocks contained in $N_{k}$ and $N_{l}$.

ii) Suppose that $A$ is not invertible and $1 \leq p<q$. The matrices $A^{p}$ and $A^{q}$ are similar if and only if

$$
A=P \operatorname{diag}\left(A^{\prime}, N\right) P^{-1}
$$

where $P$ is an arbitrary invertible matrix, $A^{\prime} \in G L_{n-k}(\mathbb{C})$ satisfies the properties given in i) and $N \in \mathcal{M}_{k}(\mathbb{C})$ satisfies $N^{p}=0_{k}$.

Remark 2. Let $A$ be such that $A^{p}$ and $A^{q}$ are similar. For every $\lambda \in \sigma(A) \backslash\{0\}$, there exist $t \in \llbracket 1, \operatorname{card}(\sigma(A)) \rrbracket$ and $k$, a divisor of $p^{t}-q^{t}$, such that $\lambda$ is a root of unity of order $k$.

To solve Eq. (1), it remains to determine the matrix $B$. This can be achieved by solving the Sylvester homogeneous equation $A^{p} X-X A^{q}=0$ and extracting the invertible solutions. However a more conceptual solution can be given. 
Proposition 2. Let $A, B \in G L_{n}(\mathbb{C})$ satisfy Eq. (1). Then $A$ is a polynomial in $A^{q}$ or in $A^{p}$. In particular, $B^{-1} A B$ and $A$ commute.

Proof. Let $f: x \in V \rightarrow x^{q}$ where $V$ is a neighborhood of $\sigma(A)$. According to Proposition [1, $i i), f$ is a holomorphic function that is one to one on $\sigma(A)$ and $f^{\prime} \neq 0$ on $\sigma(A)$. By [3, Theorem 2], $A$ is a polynomial in $A^{q}$. By symmetry, $A$ is a polynomial in $A^{p}$. Since $C^{p}=A^{q}, C$ and $A^{q}$ commute as $C$ and $A$ do.

In the next two results, we assume that $A$ is diagonalizable. Note that we will see, in Remark 4, that $A$ can be non-diagonalizable.

Definition. We say that $A \in \mathcal{M}_{n}(\mathbb{C})$ is a root of $I_{n}$ if there exists $k \in \mathbb{N}$ such that $A^{k}=I_{n}$.

Proposition 3. Let $A, B \in G L_{n}(\mathbb{C})$ satisfy $E q$ (1). If $A$ is diagonalizable, then $A$ is a root of $I_{n}$ and $B^{-1} A B$ is a power of $A$.

Proof. Using Proposition 1 with $U=\sigma(A)$, we deduce that the eigenvalues of $A$ are roots of unity such that their orders are coprime to $p q$. Since $A$ is diagonalizable, there exists $r \in \mathbb{N}$ such that $\operatorname{gcd}(r, p q)=1$ and $A^{r}=I_{n}$. Therefore there exist $\alpha, \beta \in \mathbb{Z}$ such that $\alpha p+\beta r=1$. We deduce that $C=\left(C^{p}\right)^{\alpha}\left(C^{r}\right)^{\beta}=A^{\alpha q}$.

Now we give a complete solution of $\mathrm{Eq}$ (1) in the unknowns $A, B$ when $A$ is assumed to have $n$ distinct eigenvalues.

Remark 3. Suppose that $B^{-1} A^{p} B=A^{q}$ and that the eigenvalues $\left(\lambda_{i}\right)_{i}$ of $A$ are non-zero and pairwise distinct. We may assume that $A=\operatorname{diag}\left(\lambda_{1}, \cdots, \lambda_{n}\right) . B y$ reordering the $\left(\lambda_{i}\right)_{i}$, we may assume that the permutation $\pi$, considered in Remark 1. $i i)$, is the product of disjoint cycles $\left(C_{k}\right)_{k}$ in the form

$$
C_{k}=\left(\lambda_{i_{k}}, \lambda_{i_{k}+1}, \cdots, \lambda_{i_{k+1}-1}\right) .
$$

Since $B^{-1} A B$ is diagonal, $B$ permutes the eigenspaces of $A$ and

$$
B=\operatorname{diag}\left(b_{1}, \cdots, b_{n}\right) \Sigma
$$

where the entries $\left(b_{i}\right)_{i \leq n}$ are complex numbers and $\Sigma$ is a permutation matrix. The condition $B^{-1} A^{p} B=A^{q}$ is equivalent to

$$
\Sigma^{-1} \operatorname{diag}\left(\lambda_{1}^{p}, \cdots, \lambda_{n}^{p}\right) \Sigma=\operatorname{diag}\left(\lambda_{1}^{q}, \cdots, \lambda_{n}^{q}\right) .
$$

By Proposition 1, ii), the $\left(\lambda_{i}^{p}\right)_{i}$ and the $\left(\lambda_{i}^{q}\right)_{i}$ are pairwise distinct. Therefore, $\Sigma$ is the permutation matrix associated to $\pi$. Thus the solutions in $B$ are trivial and we may suppose that $\pi$ is the cycle $(1,2, \cdots, n)$.

Notation. Denote by $R$ the $\operatorname{ring} \mathbb{Z} /\left(q^{n}-p^{n}\right)$.

Theorem 2. Let $A=\operatorname{diag}\left(\lambda_{1}, \cdots, \lambda_{n}\right)$, with the non-zero and pairwise distinct $\left(\lambda_{i}\right)^{\prime} s$, be such that $A^{p}$ and $A^{q}$ are similar. There exists

$$
k_{1} \in R \backslash \bigcup_{z \mid n, z<n}\left(\frac{q^{n}-p^{n}}{q^{z}-p^{z}}\right) R
$$

such that, for every $1 \leq u \leq n, \lambda_{u}=\exp \left(\frac{2 i \pi k_{u}}{q^{n}-p^{n}}\right)$ where $k_{u}=\left(p^{-1} q\right)^{u-1} k_{1}$.

Proof. We put $\lambda_{n+1}=\lambda_{1}$. According to Remark 1, ii),

$$
\sigma(A) \subset V_{n}=\left\{z \in \mathbb{C} \mid z^{q^{n}-p^{n}}=1\right\},
$$

that is, for all $u \leq n$, there exists $k_{u} \in R$ such that

$$
\lambda_{u}=\exp \left(\frac{2 i \pi k_{u}}{q^{n}-p^{n}}\right)
$$


The condition to be fulfilled is: for all $u \leq n, \lambda_{u}^{q}=\lambda_{u+1}^{p}$ or $q k_{u}=p k_{u+1}$. Note that $p^{-1}$, the inverse of $p$ in $R$ exists since $\operatorname{gcd}(p, q)=1$. Thus $k_{u+1}=\left(p^{-1} q\right) k_{u}$ and $k_{u}=\left(p^{-1} q\right)^{u-1} k_{1}$. Hence $\sigma(A)$ is determined by the choice of $k_{1}$. Remark that $\left(p^{-1} q\right)^{n}=1$ and then $k_{n+1}=k_{1}$.

Moreover the $\left(k_{u}\right)_{u \leq n}$ must be distinct. We consider the following equation in $R:\left(p^{-1} q\right)^{\alpha} k=k$ where $\alpha \in \llbracket 1, n \llbracket$. Since $p, q$ are invertible in $R$, we obtain $k\left(p^{\alpha}-q^{\alpha}\right)=0$. From

$$
\operatorname{gcd}\left(q^{n}-p^{n}, p^{\alpha}-q^{\alpha}\right)=q^{z}-p^{z},
$$

where $z=\operatorname{gcd}(n, \alpha)$, we deduce that $p^{\alpha}-q^{\alpha}=\left(q^{z}-p^{z}\right) f$ where $f$ is invertible in $R$. Therefore $k \in\left(\frac{q^{n}-p^{n}}{q^{z}-p^{z}}\right) R$. When $\alpha$ goes through $\{1, \cdots, n-1\}, z$ goes through the strict divisors of $n$. Finally the required condition on $k_{1}$ is

$$
\text { for every strict divisor } z \text { of } n, k_{1} \notin\left(\frac{q^{n}-p^{n}}{q^{z}-p^{z}}\right) R \text {. }
$$

Remark 4. There are invertible solutions of Eq. (1) such that $A$ is not diagonalizable and $C$ is not a power of $A$. Indeed, let $n=4, p=2, q=3$. If $A \in G L_{4}(\mathbb{C})$ is such that $A^{2}$ and $A^{3}$ are similar and if $\sigma(A)=\{\lambda, \mu\}$, then, by Remark 2 , $\lambda^{5}=\mu^{5}=1$. A solution of Eq. (1) is

$$
A=\left(\begin{array}{llll}
\lambda & 1 & 0 & 0 \\
0 & \lambda & 0 & 0 \\
0 & 0 & \bar{\lambda} & 1 \\
0 & 0 & 0 & \bar{\lambda}
\end{array}\right), B=\left(\begin{array}{cccc}
0 & 0 & 1 & 0 \\
0 & 0 & 0 & \bar{\alpha} \\
1 & 0 & 0 & 0 \\
0 & \alpha & 0 & 0
\end{array}\right)
$$

where $\lambda=e^{2 i \pi / 5}, \alpha=\frac{3}{2} e^{6 i \pi / 5}$. Clearly, $C=\left(\begin{array}{cccc}\bar{\lambda} & \alpha & 0 & 0 \\ 0 & \bar{\lambda} & 0 & 0 \\ 0 & 0 & \lambda & \bar{\alpha} \\ 0 & 0 & 0 & \lambda\end{array}\right)$ is not a power of $A$.

We consider the case where $A$ has a sole eigenvalue.

Notation. Let $S$ be a square matrix. We denote by $\mathcal{C}(S)$ the set of invertible matrices that commute with $S$, that is

$$
\mathcal{C}(S)=\left\{\Delta \in G L_{n}(\mathbb{C}) \mid \Delta S=S \Delta\right\}
$$

Remark 5. It should be noted that $\mathcal{C}(S)$ is not the commutant of $S$.

Proposition 4. Let $A \in G L_{n}(\mathbb{C})$ such that $\sigma(A)=\{\lambda\}$ with $\lambda^{q-p}=1$ and let $N=A-\lambda I_{n}$. One has

i) $A^{p}$ and $A^{q}$ are similar.

ii) There exists a unique polynomial $P \in \mathbb{C}[X]$ with least degree, such that for every matrix $C$ defined above, $C-\lambda I_{n}=P(N)$.

iii) There exists a matrix $B_{0}$ such that $B_{0}^{-1} N B_{0}=P(N)$ and the set of matrices $B$ satisfying $B^{-1} A^{p} B=A^{q}$ is $\mathcal{C}(N) B_{0}$.

Proof. $i$ ) is clear.

ii) Necessarily $C$ is in the form $C=\lambda I_{n}+M$ where $M$ is a nilpotent matrix, similar to $N$ and such that $M N=N M$. Let $d$ be the nilpotence index of $N$ or $M$. The relation $C^{p}=A^{q}$ can be written

$$
\left(\sum_{k=2}^{d}\left(\begin{array}{c}
p \\
k-1
\end{array}\right) \lambda^{p-k+1} M^{k-2}\right) M=\left(\sum_{k=2}^{d}\left(\begin{array}{c}
q \\
k-1
\end{array}\right) \lambda^{q-k+1} N^{k-2}\right) N,
$$

that is $E M=F N$ where $E, F$ are invertible matrices that both commute with $M$ and $N$. According to Proposition 2, $A$ is a polynomial in $A^{q}=C^{p}$. Therefore $N$ 
is a polynomial in $C^{p}$ and $N$ is a polynomial in $M$. Since $N$ and $M$ are nilpotent and $\operatorname{ker}(M)=\operatorname{ker}(N)$, one has $N=\beta_{1} M+\beta_{2} M^{2}+\cdots+\beta_{d-1} M^{d-1}$ where $\beta_{1} \neq 0$. By [3. Theorem 2], we conclude that $M$ is a polynomial in $N$ in the form

$$
M=P(N)=\alpha_{1} N+\alpha_{2} N^{2}+\cdots+\alpha_{d-1} N^{d-1}
$$

where $\alpha_{1} \neq 0$. Since $N, N^{2}, \cdots, N^{d-1}$ are linearly independent, we can determine the $\left(\alpha_{i}\right)_{i=1, \cdots, d-1}$. Finally we obtain a unique solution in $M$ as a function of $\lambda$ and $N$.

iii) It remains to determine the matrices $B$ such that

$$
B^{-1} N B=M \text {. }
$$

First we build a particular solution of (4). Let $\left\{e_{1}, \cdots, e_{n}\right\}$ be a basis in which $N$ is in Jordan form: $N=\operatorname{diag}\left(J_{r_{1}}, \cdots, J_{r_{k}}\right)$ where $J_{r_{i}}$ is the Jordan nilpotent block of dimension $r_{i}$. The matrix $M$ is a polynomial in $N$, hence $M=\operatorname{diag}\left(M_{1}, \cdots, M_{k}\right)$ where $M_{i}$ has dimension $r_{i}$. We look for a particular solution in the form

$$
B_{0}=\operatorname{diag}\left(B_{1}, \cdots, B_{k}\right)
$$

where, for every $i, B_{i}^{-1} J_{r_{i}} B_{i}=M_{i}$. Thus we may assume that $N=J_{n}$. The set $\mathcal{B}=\left\{M^{n-1}\left(e_{n}\right), M^{n-2}\left(e_{n}\right), \cdots, e_{n}\right\}$ is a basis of $\mathbb{C}^{n}$. Let $P$ be the $\mathcal{B}$-basis matrix. We have $M=P N P^{-1}$ and we can choose $B_{0}=P^{-1}$. Clearly the set of solutions of (41) is $\mathcal{C}(N) B_{0}$.

Remark 6. i) A complete description of $\mathcal{C}(N)$ is given in 2 or 9 .

ii) Knowing $A$, all the computations can be performed explicitly.

3. On the Equation $A^{r} B^{s} A^{r^{\prime}} B^{s^{\prime}}=\epsilon I_{2}$

Instead of Eq. (11), we consider the more general matrix equation

$$
A^{r} B^{s} A^{r^{\prime}} B^{s^{\prime}}=\epsilon I_{2} \text {. }
$$

We restrict ourselves to $2 \times 2$ complex matrices. The integers $r, r^{\prime}$ (resp. $s, s^{\prime}$ ) are considered to be coprime and $\epsilon= \pm 1$.

Definition. The complex matrices $A, B$ are said simultaneously triangularizable (denoted by $S T$ ) if there exists an invertible complex matrix $P$ such that $P^{-1} A P$ and $P^{-1} B P$ are upper triangular.

Lemma 2. We may assume that the matrices $A, B$ satisfy $\operatorname{det}(A)=\operatorname{det}(B)=1$.

Proof. Let $A, B$ be invertible matrices satisfying Eq (2). There exist $\lambda, \mu \in \mathbb{C}^{*}$ such that $A=\lambda A_{1}$ with $\operatorname{det}\left(A_{1}\right)=1$ and $B=\mu B_{1}$ with $\operatorname{det}\left(B_{1}\right)=1$. One has $\lambda^{2 r+2 r^{\prime}} \mu^{2 s+2 s^{\prime}}=1$, that is $\lambda^{r+r^{\prime}} \mu^{s+s^{\prime}}= \pm 1$. Therefore

$$
A_{1}^{r} B_{1}^{s} A_{1}^{r^{\prime}} B_{1}^{s^{\prime}}=\lambda^{r+r^{\prime}} \mu^{s+s^{\prime}}\left(\epsilon I_{2}\right)= \pm \epsilon I_{2} .
$$

In the sequel, we assume that $\operatorname{det}(A)=\operatorname{det}(B)=1$. The case where $A$ and $B$ are $\mathrm{ST}$ is not interesting. Indeed we have

Proposition 5. Suppose that $A, B$ are ST. The Eq. (2) can be reduced to a triangular system of polynomial equations in four unknowns.

Proof. We may assume that $A, B$ are upper triangular matrices in the form

$$
A=\left(\begin{array}{cc}
u & v \\
0 & 1 / u
\end{array}\right), B=\left(\begin{array}{cc}
p & q \\
0 & 1 / p
\end{array}\right),
$$


where $(p, u, q, v) \in\left(\mathbb{C}^{*}\right)^{2} \times \mathbb{C}^{2}$. One checks easily that Eq. (2) is equivalent to a system in the form

$$
\left\{\begin{array}{cl}
u^{r+r^{\prime}} p^{s+s^{\prime}} & =\epsilon \\
v \phi(u, p)+q \psi(u, p) & =0
\end{array}\right.
$$

where $\phi, \psi$ are polynomials that depend on $r, r^{\prime}, s, s^{\prime}$.

In the sequel, we assume that $A, B$ are not $S T$, that is they have no common eigenvectors.

Lemma 3. Let $A, B \in \mathcal{M}_{2}(\mathbb{C})$ be not $S T$. They are simultaneously similar to two symmetric matrices.

Proof. Case 1. $A$ is diagonalizable. We may asssume that $A$ is diagonal and $B=\left(\begin{array}{ll}a & b \\ c & d\end{array}\right)$ with $b c \neq 0$. Write $P=\left(\begin{array}{ll}x & 0 \\ 0 & y\end{array}\right)$, with $x^{2}=b, y^{2}=c$. The matrices $P^{-1} A P$ and $P^{-1} B P$ are symmetric.

Case 2. $A, B$ are not diagonalizable. One has $A=\lambda I_{2}+M, B=\mu I_{2}+N$ where $\lambda, \mu \in \mathbb{C}$ and $M, N$ are nilpotent matrices that are not $S T$. We can construct a basis of $\mathbb{C}^{2}$ containing an eigenvector of $M$ and an eigenvector of $N$. Thus we may assume that

$$
A=M=\left(\begin{array}{ll}
0 & \alpha \\
0 & 0
\end{array}\right) \text { and } B=N=\left(\begin{array}{ll}
0 & 0 \\
\beta & 0
\end{array}\right),
$$

with $\alpha, \beta \in \mathbb{C}-\{0\}$. For $P=\left(\begin{array}{cc}1 & i \\ 1 & -i\end{array}\right), P^{-1} A P$ and $P^{-1} B P$ are symmetric.

Corollary 1. Let $A, B \in \mathcal{M}_{2}(\mathbb{C})$ that are not $S T$. If $A^{r} B^{s} A^{r^{\prime}} B^{s^{\prime}}=\epsilon I_{2}$, then $A^{-r} B^{-s} A^{-r^{\prime}} B^{-s^{\prime}}=\epsilon I_{2}$.

Proof. By Lemma 3. we may assume that $A, B$ are symmetric matrices. Easy computation allows to conclude the assertion.

Proposition 6. Let $A, B \in S L_{2}(\mathbb{C})$ that are not $S T$ and satisfy Eq. (2). Then

$$
\left(A^{r-r^{\prime}}= \pm I_{2} \text { or } B^{s}= \pm I_{2}\right) \text { and }\left(B^{s-s^{\prime}}= \pm I_{2} \text { or } A^{r^{\prime}}= \pm I_{2}\right) \text {. }
$$

Proof. By Corollary 1, we find easily $A^{-r} B^{-s} A^{-r^{\prime}}=A^{-r^{\prime}} B^{-s} A^{-r}$, that is $A^{r-r^{\prime}} B^{s}=$ $B^{s} A^{r-r^{\prime}}$. Since $B^{s}$ and $A^{r-r^{\prime}}$ commute, they have a common eigenvector. That is impossible but if $B^{s}= \pm I_{2}$ or $A^{r-r^{\prime}}= \pm I_{2}$. By symmetry, the second relation holds.

Notation. Let $k \in \mathbb{Z}$. Denote by $\phi_{k}$ the continuous function defined on $\mathbb{C}^{*}$ by

$$
\phi_{k}(t)=\left\{\begin{array}{cl}
\frac{1-t^{2 k}}{t^{k-1}\left(1-t^{2}\right)} & \text { if } t \neq \pm 1 \\
k & \text { if } t=1 \\
(-1)^{k-1} k & \text { if } t=-1 .
\end{array}\right.
$$

Lemma 4. Let $A, B \in S L_{2}(\mathbb{C})$ that are not $S T$. The following assertions hold i) The matrices $A, B$ are simultaneously similar to matrices in the form

$$
A_{1}=\left(\begin{array}{cc}
u & v \\
0 & u^{-1}
\end{array}\right), B_{1}=\left(\begin{array}{cc}
p & 0 \\
q & p^{-1}
\end{array}\right),
$$

where $u, v, p, q$ are non-zero complex numbers such that

$$
\left(p^{2}-1\right)\left(u^{2}-1\right)+u v p q \neq 0 .
$$

ii) For every $k \in \mathbb{Z}$, we have

$$
A_{1}^{k}=\left(\begin{array}{cc}
u^{k} & v \phi_{k}(u) \\
0 & u^{-k}
\end{array}\right), B_{1}^{k}=\left(\begin{array}{cc}
p^{k} & 0 \\
q \phi_{k}(p) & p^{-k}
\end{array}\right) .
$$


iii) Let $\alpha \in\{1,-1\}$. The equality $A_{1}^{k}=\alpha I_{2}$ (resp. $B_{1}^{k}=\alpha I_{2}$ ) holds if and only if $u^{k}=\alpha$ and $u^{2} \neq 1$ (resp. $p^{k}=\alpha$ and $p^{2} \neq 1$ ).

Proof. $i$ ) There exists a basis of $\mathbb{C}^{2}$ containing an eigenvector of $A$ and an eigenvector of $B$. Such a change of basis transforms $A$ and $B$ in the form

$$
A_{1}=\left(\begin{array}{cc}
u & v \\
0 & u^{-1}
\end{array}\right), B_{1}=\left(\begin{array}{cc}
p & 0 \\
q & p^{-1}
\end{array}\right)
$$

where $u v p q \neq 0$. Moreover $A_{1}$ and $B_{1}$ are not $S T$ is equivalent to $\operatorname{det}\left(\left[A_{1}, B_{1}\right]\right) \neq 0$, that is Relation (5).

ii) and iii) are straightforward computations.

Lemma 5. Let $A, B \in \mathcal{M}_{2}(\mathbb{C})$. The matrices $A, B$ have determinant 1 , are not $S T$ and $(A B)^{2}=-I_{2}$ if and only if $A, B$ are simultaneously similar to the matrices $A_{1}, B_{1}$ defined in Lemma 4 with the condition

$$
q v=\frac{-1-u^{2} p^{2}}{u p}, \text { where }\left\{\begin{array}{l}
u p \neq 0 \\
u^{2}+p^{2} \neq 0 \\
1+u^{2} p^{2} \neq 0 .
\end{array}\right.
$$

Proof. This follows easily from Lemma 4, $i$ ).

Proposition 7. Let $A, B \in S L_{2}(\mathbb{C})$ that are not $S T$. The matrices $A, B$ satisfy Eq. (2) if and only if there exists $\alpha \in\{-1,1\}$ such that

$$
A^{r-r^{\prime}}=\alpha I_{2}, B^{s-s^{\prime}}=-\alpha \epsilon I_{2} \text { and }\left(A^{r} B^{s}\right)^{2}=-I_{2} .
$$

Proof. According to Proposition 6, we have to consider the following cases.

Case 1. $A^{r-r^{\prime}}= \pm I_{2}$ and $A^{r^{\prime}}= \pm I_{2}$. This implies that $A^{r}= \pm I_{2}$. Since $\operatorname{gcd}\left(r, r^{\prime}\right)=1, A= \pm I_{2}$, that is impossible.

Case 2. $B^{s}= \pm I_{2}$ and $B^{s-s^{\prime}}= \pm I_{2}$. By symmetry of case 1 .

Case 3. $B^{s}= \pm I_{2}$ and $A^{r^{\prime}}= \pm I_{2}$. One has $A^{r} B^{s^{\prime}}= \pm I_{2}$. Then, for instance, $A^{r}= \pm I_{2}$ and finally $A= \pm I_{2}$. This is impossible.

Case 4. There exist $\alpha, \beta \in\{-1,1\}$ such that $A^{r-r^{\prime}}=\alpha I_{2}$ and $B^{s-s^{\prime}}=\beta I_{2}$. One has $\left(A^{r} B^{s}\right)^{2}= \pm I_{2}$. Assume $\left(A^{r} B^{s}\right)^{2}=I_{2}$. The relations $\operatorname{det}(A)=\operatorname{det}(B)=1$ imply $A^{r} B^{s}= \pm I_{2}$. Then, for instance $A^{r}= \pm I_{2}$ and we conclude as in Case 3 . Finally $\left(A^{r} B^{s}\right)^{2}=-I_{2}$ and $\beta=-\alpha \epsilon$.

The converse is clear.

Corollary 2. If $r-r^{\prime}= \pm 1$ or $s-s^{\prime}= \pm 1$, then there are no pairs $(A, B) \in S L_{2}(\mathbb{C})$ that are not ST and satisfy Eq. (2).

Proof. Assume, for instance, that $r-r^{\prime}= \pm 1$. According to Proposition 7 necessarily one has $A= \pm I_{2}$, that is impossible.

Theorem 3. Assume that $r-r^{\prime} \neq \pm 1$ and $s-s^{\prime} \neq \pm 1$. A pair $(A, B) \in S L_{2}{ }^{2}(\mathbb{C})$ is not ST and satisfies Eq. (2) if and only if it is conjugate to a pair in the form

$$
\left(\left(\begin{array}{cc}
u & v \\
0 & u^{-1}
\end{array}\right),\left(\begin{array}{cc}
p & 0 \\
q & p^{-1}
\end{array}\right)\right)
$$

where $u, v, p, q \in \mathbb{C}^{*}$ are such that

$$
\begin{gathered}
u^{r-r^{\prime}}= \pm 1, p^{s-s^{\prime}}=-\epsilon u^{r-r^{\prime}} \\
u^{2 r}+p^{2 s} \neq 0,1+u^{2 r} p^{2 s} \neq 0 \text { and } \\
q v=\frac{-1-u^{2 r} p^{2 s}}{u^{r} \phi_{r}(u) p^{s} \phi_{s}(p)} .
\end{gathered}
$$


Proof. According to Lemma 4, we may assume $A=\left(\begin{array}{cc}u & v \\ 0 & u^{-1}\end{array}\right), B=\left(\begin{array}{cc}p & 0 \\ q & p^{-1}\end{array}\right)$, where $u v p q \neq 0$. For every $r \in \mathbb{Z}$, one has $A^{r}=\left(\begin{array}{cc}u^{r} & v \phi_{r}(u) \\ 0 & u^{-r}\end{array}\right), B^{s}=\left(\begin{array}{cc}p^{s} & 0 \\ q \phi_{s}(p) & p^{-s}\end{array}\right)$. According to Proposition [7. $\left(A^{r} B^{s}\right)^{2}=-I_{2}$. Suppose that $A^{r}$ and $B^{s}$ have a common eigenvector. Then, for instance, $A^{r}= \pm I_{2}, A^{r^{\prime}}= \pm I_{2}$ and finally $A= \pm I_{2}$. This is impossible. Thus $A^{r}$ and $B^{s}$ are not $S T$. By Lemma 5 , Conditions (7) and (8) hold. By Proposition 7 there exists $\alpha \in\{-1,1\}$ such that $A^{r-r^{\prime}}=\alpha I_{2}, B^{s-s^{\prime}}=-\alpha \epsilon I_{2}$. We deduce that $u^{r-r^{\prime}}=\alpha$ and $p^{s-s^{\prime}}=-\alpha \epsilon$.

The converse is clear.

Remark 7. Note that $u$ and $p$ are roots of unity except if $r=r^{\prime}= \pm 1$ or $s=s^{\prime}= \pm 1$.

Acknowledgements. The author thanks F. Luca for his participation in the proof of Proposition 1 and D. Adam for many valuable discussions. The author thanks the referees for their valuable suggestions.

\section{REFERENCES}

[1] J. Benitez, N. Thome. $\{k\}$-group periodic matrices. SIAM J. Matrix Anal. Applic. 28.1 (2006) 9-25.

[2] F. Gantmacher. The theory of matrices I. Chelsea, New York, 1959.

[3] R. Horn, G. Piepmeyer. Two applications of the theory of primary matrix functions. Linear Algebra and its Applications, 361 (2003) 99-106.

[4] L. Lebtahi, O. Romero, N. Thome. Characterization of $\{K, s+1\}$-potent matrices and applications. Linear Algebra and its Applications, 436 (2012) 293-306.

[5] L. Lebtahi, O. Romero, N. Thome. Relations between $\{K, s+1\}$-potent matrices and different classes of complex matrices. To appear in Linear Algebra and its Applications, DOI:10.1016/j.laa.2011.10.042.

[6] R. Loewy, V. Mehrmann. A note on Potter's theorem for quasi-commutative matrices. Linear Algebra and its Applications, 430 (2009) 1812-1825.

[7] W.F. Trench. Characterization and properties of matrices with $k$-involutory symmetries. Linear Algebra and its Applications, 429 (2008) 2278-2290.

[8] W.F. Trench. Characterization and properties of matrices with k-involutory symmetries II. Linear Algebra and its Applications, 432 (2010) 2782-2797.

[9] H. Turnbull, A. Aitken. An introduction to the theory of canonical matrices. Dover, New York, 1961.

GÉrald Bourgeois, GAATI, Université de la polynésie française, BP 6570, 98702 FAA'A,

Tahiti, Polynésie Française.

E-mail address: bourgeois.gerald@gmail.com 